\title{
A Fluorometric Bio-Sniffer (Bio-chemical Gas Sensor) with UV-LED for Monitoring Gaseous Formaldehyde
}

T. Gessei ${ }^{1,2}$, Gen Itabashi $^{1}$, D. Takahashi ${ }^{1}$, T. Arakawa $^{1}$, H. Kudo ${ }^{1}$, K. Mitsubayashi $^{1 \dagger}$

${ }^{1}$ Institute of Biomaterials and Bioengineering, Tokyo Medical and Dental University, 2-3-10 KandaSurugadai, Chiyoda-ku, Tokyo 101-0062, Japan;

2 Tokyo Metropolitan Industrial Technology Research Institute, Tokyo, Japan, 3-13-10 Nishigaoka, Kita-ku, Tokyo 115-8586, Japan

\section{INTRODUCTION}

Gaseous formaldehyde is one of the harmful and injurious VOCs (volatile organic compounds), and has been reported to develop Sick-house syndrome, especially at the hermetic house. In this work, we have constructed a high-sensitive $\mathrm{NADH}(\lambda=340 \mathrm{~nm})$ fluorometric biochemical-sniffer by incorporating fibre-optic device with UV-LED $(\lambda=335 \mathrm{~nm})$ based excitation system and FALDH (formaldehyde dehydrogenase) enzyme membrane into a diaphragm reaction cell with gas- and liquid-compartments for the formaldehyde monitoring in the gas phase.

\section{EXPERIMENTAL}

The NADH fluorometric sensor was constructed with an UV-LED ( $\lambda=335 \mathrm{~nm}$, Figure 1), a fibre optic spectrometer, and an optical fibre probe. The UV-LED light source and the spectrometer (or photomultiplier tube) were connected to the optical fibre probe by Y-shaped optical fibre. A band-pass filter (BPF:340nm) and a long-pass filter $(\mathrm{Cf}=400 \mathrm{~nm})$ were placed. The FALDH immobilized membrane was attached on the optical fibre probe as the separating diaphragm between the gas- and liquidcompartments. Measurement of the gaseous formaldehyde concentration was carried out by PB (w/ $\mathrm{NAD+}$ ) rinsing to the liquid compartment with the optical probe. The excitation UV light was conducted to the sensing terminal of the optical fibre probe. Various concentrations of gaseous formaldehyde were supplied from a gas generator to the gas-compartment. The fluorescent signals of NADH, produced by enzymatic reaction of FALDH, were then guided to the spectrometer (or photomultiplier tube) and recorded using a laptop PC..

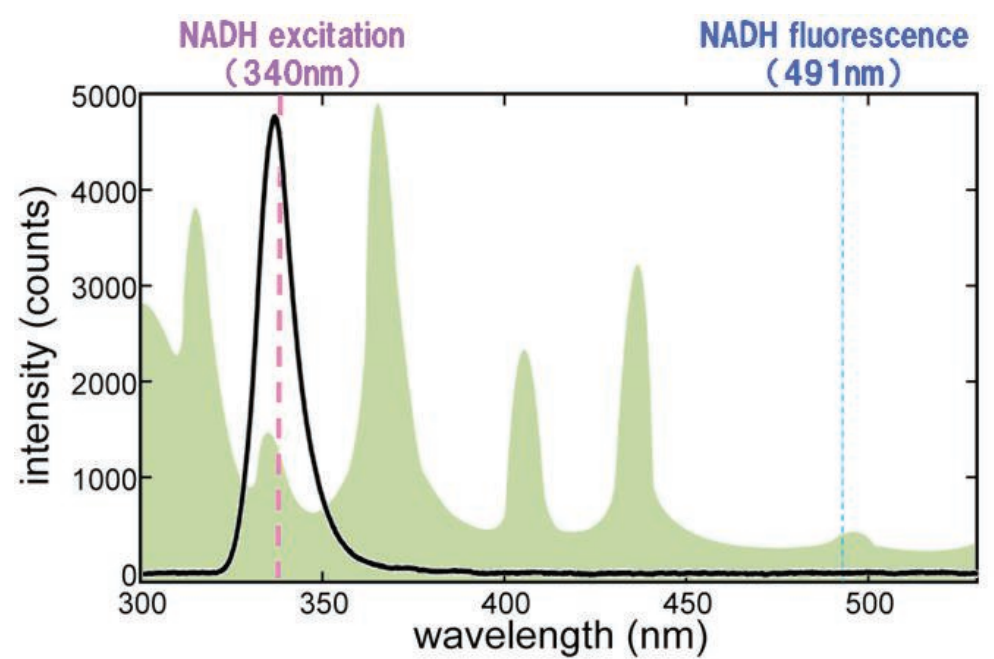

Figure 1. Comparison of light spectrum between UV-LED and Mercury lamps.

\section{RESULTS AND DISCUSSION}

The change of fluorescent intensity induced NADH generation was observed by the application of formaldehyde vapour (Figure 2). The peak wavelength of the fluorescence was $491 \mathrm{~nm}$. The fluorescent intensity of the bio-sniffer was related to the concentration of the gaseous formaldehyde (lower detection limit: $3.0 \mathrm{ppb}$ ). The fluorometric bio-sniffer was possible to monitor the concentration change of formaldehyde vapour with good sensitivity and ultrahigh gas-selectivity. 


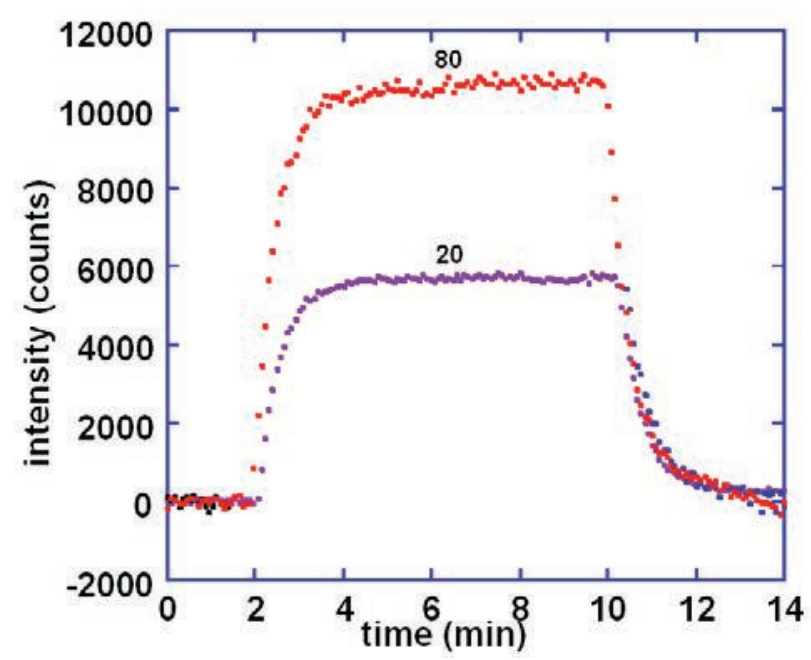

Figure 2. Typical responses of fluorometric bio-sniffer for formaldehyde (20 and $80 \mathrm{ppb})$ in the gas phase.

\section{ACKNOWLEDGEMENTS}

This work was partly supported by Japan Society for the Promotion of Science, by Japan Science and Technology Agency and by MEXT "Advanced Research Program in Sensing Biology".

\section{REFERENCES}

[1] H. Kudo, et.al., A NADH-dependent fiber-optic biosensor for ethanol determination with a UV-LED excitation system, Sensors and Actuators, (2009), B 141, 20-25.

[2] K. Mitsubayashi, et.al., Gas-Phase Biosensor for Ethanol, Anal. Chem., (1994), 66, 3297-3302. 